\title{
Nanometer-size magnetic domains and coherent magnetization reversal in a giant exchange-bias system
}

\author{
C. Dufour, ${ }^{1,}{ }^{*}$ M. R. Fitzsimmons, ${ }^{2, \dagger}$ J. A. Borchers,${ }^{3}$ M. Laver, ${ }^{4,5,6}$ K. L. Krycka, ${ }^{3}$ K. Dumesnil, ${ }^{1}$ S. M. Watson, ${ }^{3}$ \\ W. C. Chen, ${ }^{3,7}$ J. Won, ${ }^{2}$ and S. Singh ${ }^{2,8}$ \\ ${ }^{1}$ Laboratoire de Physique des Matériaux, Université H. Poincaré Nancy I, BP 239, 54506 Vandouvre les Nancy Cedex, France \\ ${ }^{2}$ Los Alamos National Laboratory, Los Alamos, New Mexico 87545, USA \\ ${ }^{3}$ National Institute of Standards and Technology, Gaithersburg, Maryland 20899, USA \\ ${ }^{4}$ Laboratory for Neutron Scattering, Paul Scherrer Institut, CH 5232 Villigen, Switzerland \\ ${ }^{5}$ Materials Research Division, Ris $\varnothing$ DTU, Technical University of Denmark, DK-4000 Roskilde, Denmark \\ ${ }^{6}$ Nano-Science Center, Niels Bohr Institute, University of Copenhagen, DK-2100 Kфbenhavn, Denmark \\ ${ }^{7}$ University of Maryland, College Park, Maryland 20742, USA \\ ${ }^{8}$ Solid State Physics Division, Bhabha Atomic Research center, Mumbai 400085, India
}

(Received 3 May 2011; revised manuscript received 22 June 2011; published 23 August 2011)

\begin{abstract}
The role of magnetic domains and domain walls in exchange bias has stimulated much contemporary deliberation. Here we present compelling evidence obtained with small-angle scattering of unpolarized- and polarized-neutron beams that magnetization reversal occurs via formation of 10-100s nm-sized magnetic domains in an exchange-biased $\mathrm{DyFe}_{2} / \mathrm{YFe}_{2}$ superlattice. The reversal mechanism is observed to involve rotation of magnetization in and out of the sample plane. Remarkably, the domains are arranged in a quasiperiodic manner in the plane of the sample. The length scale of domain formation is similar to that of structural defects at the seed-layer-superlattice interface.
\end{abstract}

DOI: 10.1103/PhysRevB.84.064420

PACS number(s): 75.70.Cn, 75.60.Jk, 75.30.Et, 61.05.fg

\section{INTRODUCTION}

Exchange coupling plays an important role in magnetic materials technology as a means to inhibit the response of a magnetic material to a changing magnetic field. For example, pinned magnetization can lead to a high product of magnetization and field in the second quadrant of the hysteresis loop, which is an important figure of merit for permanent magnets. ${ }^{1}$ A second example is exchange bias-the shift of the magnetization of a ferromagnet (FM) $B_{\mathrm{EB}}$ about zero applied field - which can be observed when unpinned spins of a FM are coupled to the pinned uncompensated magnetization of an antiferromagnet (AF) or a ferrimagnet (FI). ${ }^{2-19}$ Exchange bias has important technological applications in "spin-valve" devices, ${ }^{20}$ which are based on a switch fabricated from two FM layers - one pinned (by exchange bias) and the other unpinned. In technological applications where pinned magnetization is important, a better understanding of the magnetization reversal process, in particular how pinning of magnetization is compromised, should enable development of more robust materials.

In exchange-coupled multilayers, a unidirectional anisotropy resulting in exchange bias is typically promoted by cooling the sample in an applied field. Here we consider cooling fields $\mathrm{B}_{\mathrm{CF}}$ applied in the plane of $\mathrm{DyFe}_{2} / \mathrm{YFe}_{2}$ multilayers (in-plane exchange bias), and $+\mathrm{B}_{\mathrm{CF}}$ sets the positive direction of fields B applied thereafter. Previously we developed a simple one-dimensional spin-chain model for this system in which the layers are hard and soft FIs, respectively. ${ }^{21}$ Two mechanisms for magnetization reversal were identified from the model. One occurred via an exchange-spring mechanism. ${ }^{1}$ As the applied field was reduced from positive to zero, the magnetization (in the $\mathrm{YFe}_{2}$ layers) would rotate perpendicular to the applied field in a clockwise (+) or counterclockwise (-) fashion. This coherent rotation of magnetization is associated with so-called $\sigma^{ \pm}$ domains. ${ }^{22,23}$ The second mechanism involved a flipping of the magnetization of domains at the exchange bias field; in these so-called $\sigma^{0}$ domains, the magnetization would be pinned primarily along the direction of the cooling field before reaching the exchange bias field $\mathrm{B}_{\mathrm{EB}}$. Once the applied field equaled the exchange bias field, the magnetization within the $\mathrm{YFe}_{2}$ would abruptly change. With equal proportions of $\sigma^{+}$, $\sigma^{-}$, and $\sigma^{0}$ domains, the model explained all the experimental data including the magnitude and sign of the exchange bias $\left(\mathrm{B}_{\mathrm{EB}}=-2 \mathrm{~T}\right.$ at $\left.12 \mathrm{~K}\right)$, the response of the $\mathrm{YFe}_{2}$ layers to field, and the lack of response of the $\mathrm{DyFe}_{2}$ layers to field as observed using the chemical specificity of $\mathrm{x}$-ray circular dichroism for modest cooling fields. ${ }^{21}$

Direct experimental evidence has been lacking for the existence of magnetic domains, e.g., the $\sigma^{ \pm}$and $\sigma^{0}$ domains, in the $\mathrm{DyFe}_{2} / \mathrm{YFe}_{2}$ superlattice. Our previous polarizedneutron reflectometry experiments ${ }^{21}$ were unable to discern any neutron-spin-dependent off-specular reflectivity from magnetic domains on micron-size length scales. In this paper we report direct observations of nm-size domains using small angle neutron scattering (SANS). ${ }^{24,25}$

\section{SAMPLE PREPARATION AND CHARACTERIZATION}

\section{A. Sample growth}

A 50-nm-thick single crystal of (110) $\mathrm{Nb}$ was grown using molecular beam epitaxy on a double-side-polished 5-cm-diameter 0.3-mm-thick (1120) single-crystal sapphire substrate. Additional buffer layers of $\mathrm{Fe}(1.5 \mathrm{~nm})$ and $\mathrm{YFe}_{2}$ were epitaxially grown. Next 22 single crystalline bilayers were grown beginning with $\mathrm{DyFe}_{2}$ and followed by $\mathrm{YFe}_{2}$. This process yielded a [110] $\mathrm{YFe}_{2} /\left[\mathrm{DyFe}_{2} / \mathrm{YFe}_{2}\right]_{22}$ Laves phase superlattice. The $\mathrm{DyFe}_{2}$ and $\mathrm{YFe}_{2}$ layers were 


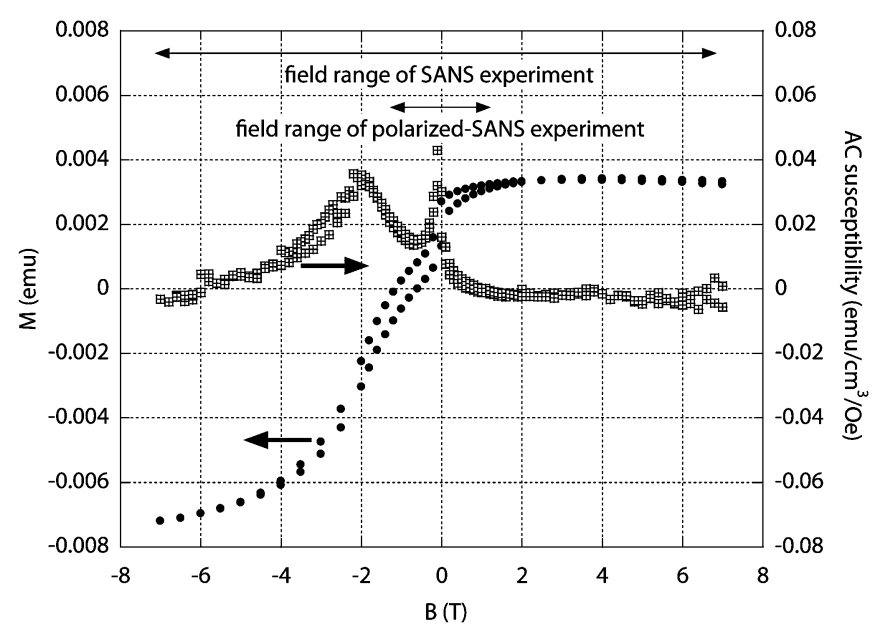

FIG. 1. Magnetization plotted as a function of applied field (solid symbols) and AC susceptibility (open symbols) at $12 \mathrm{~K}$ after field cooling in $+1 \mathrm{~T}$.

$(3.5 \pm 0.2) \mathrm{nm}$ and $(10.5 \pm 0.2) \mathrm{nm}$ thick, respectively. After growth, the wafer was cut into eight $1 \mathrm{~cm}$ by $1 \mathrm{~cm}$ samples.

\section{B. Establishing exchange bias and magnetometry}

The following procedure was employed to establish exchange bias. The samples were magnetized at room temperature in a field $\mathrm{B}=+7 \mathrm{~T}$ applied parallel to [110] for the unpolarized-beam experiment (and $+1 \mathrm{~T}$ for the polarizedbeam experiment). ${ }^{26}$ The field was reduced to $\mathrm{B}_{\mathrm{CF}}=+1 \mathrm{~T}$, and the samples cooled to $12 \mathrm{~K}$. $\mathrm{B}_{\mathrm{CF}}$ was chosen to be large enough to align the Fe spins, but not too large so as to pin a component of the $\mathrm{DyFe}_{2}$ spins along [001] (perpendicular to $\mathrm{B}_{\mathrm{CF}}$ ). ${ }^{27}$ This favors the antiparallel arrangement of magnetizations from the $\mathrm{DyFe}_{2}$ (dominated by Dy) and $\mathrm{YFe}_{2}$ (dominated by $\mathrm{Fe}$ ) layers. From magnetometry (Fig. 1) the resulting exchange bias is $\mathrm{B}_{\mathrm{EB}}=(-1.8 \pm 0.1) \mathrm{T}$. The error of $\mathrm{B}_{\mathrm{EB}}$ represents the standard deviation of measurements from four pieces taken from the middle and edges of the sample. The rapid change of magnetization at $\mathrm{B}=0$ (and sharp increase of the $\mathrm{AC}$ susceptibility at this field) is consistent with the abrupt reversal of two $\mathrm{YFe}_{2}$ layers (or $\sim 10 \%$ of the sample). This feature has not been as pronounced in previously studied samples. ${ }^{21}$

\section{Transmission electron microscopy}

Cross-sectional transmission electron microscopy (Fig. 2) shows the first few deposited bilayers of the superlattice are not coalesced. The first layer of $\mathrm{YFe}_{2}$ material consists of islands. Note the size and quasiperiodic arrangement of islands parallel to the substrate are of order $100+\mathrm{nm}$. Voids between the islands will provide a source of nuclear and magnetic contrast that may give rise to SANS and moreover pin magnetic domain walls. The layers deposited immediately after the islands form "bridge-like" structures. The superlattice is completely coalesced after deposition of about eight bilayers.

\section{Neutron and $x$-ray reflectometry}

Previously we did not observe significant diffuse scattering of polarized-neutron beams in reflection geometry at $250 \mathrm{~K}^{27}$ or $12 \mathrm{~K}^{21}$ However, in the range between 200 and $130 \mathrm{~K}$ very pronounced broadening of the specular beam, possibly off-specular scattering, was observed during the experiment reported in Ref. 27. In order to ascertain whether the origin of the diffuse scattering between 200 and $130 \mathrm{~K}$ was magnetic or structural, we measured the width of a specularly reflected $\mathrm{X}$-ray beam $\left(\mathrm{CuK}_{\alpha}\right.$ radiation) as a function of temperature (Fig. 3). The specular X-ray reflection broadens significantly below $250 \mathrm{~K}$ and then becomes narrow below $150 \mathrm{~K}$. Since the $\mathrm{X}$-ray experiment is only sensitive to the structural quality of the sample, this measurement combined with our earlier observations of spin dependence and diffuse scattering of neutron beams in the same temperature range suggest a relationship may exist between the sample's structure and magnetic behavior (e.g., through magnetoelastic coupling, which is strong in Laves phase rare-earth compounds ${ }^{28}$ ). This relationship can be further probed with SANS.

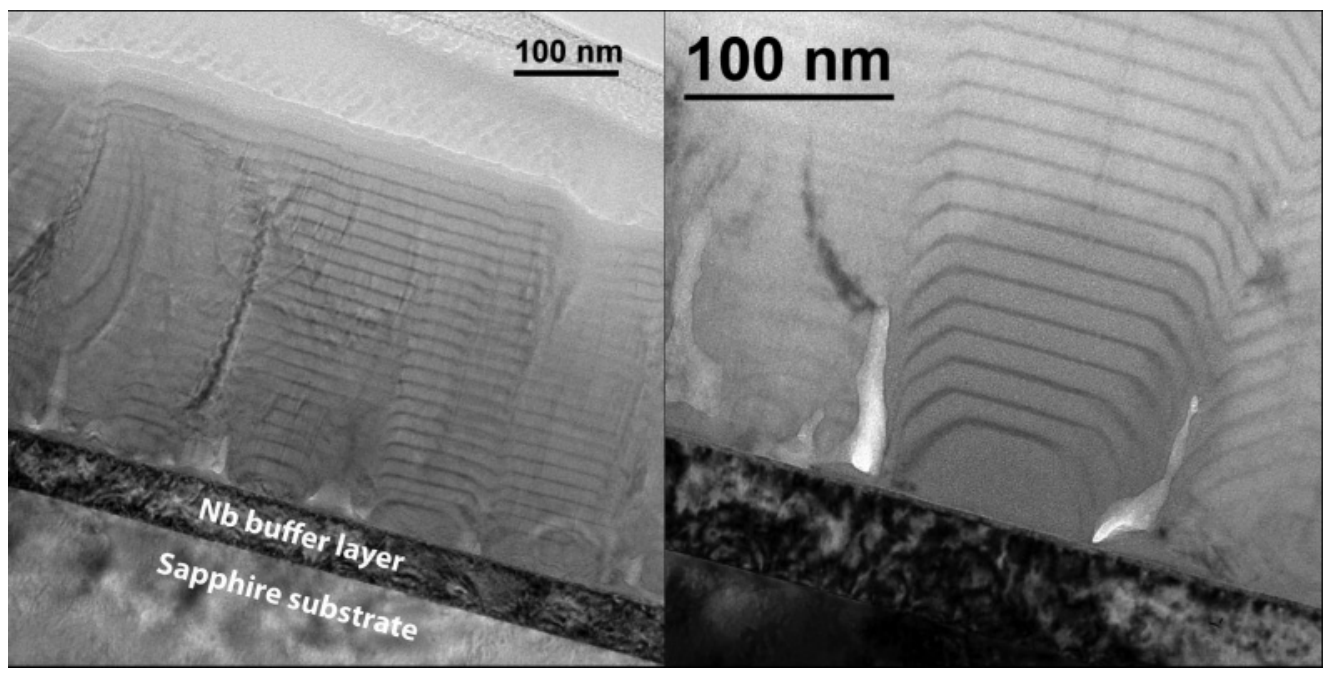

FIG. 2. Cross-sectional transmission electron micrographs of the superlattice sample, Nb buffer layer, and substrate. 


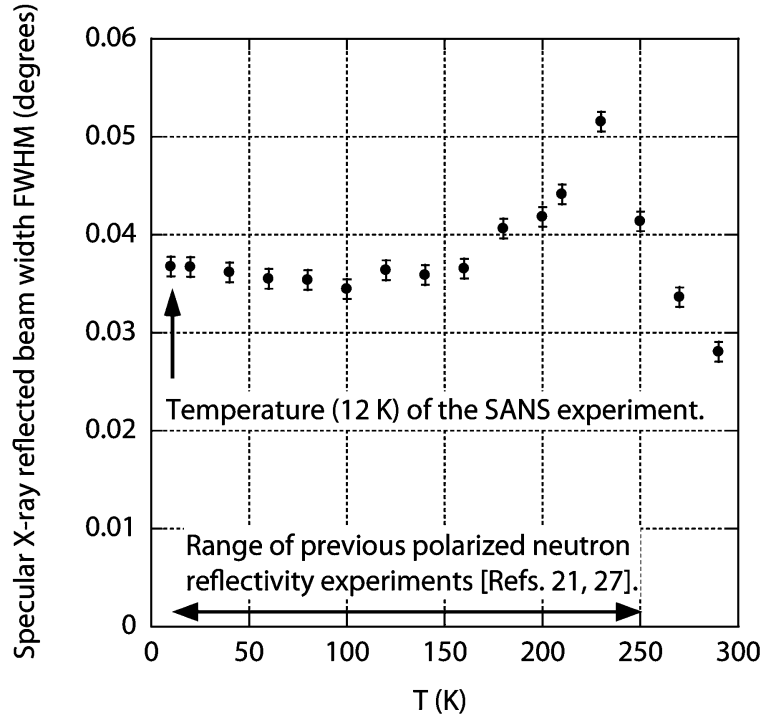

FIG. 3. Full width at half maximum of the specularly reflected $\mathrm{x}$-ray beam from the $\mathrm{DyFe}_{2} / \mathrm{YFe}_{2}$ sample as a function of temperature. (Error represents the resolution of the instrument.)

\section{SANS WITH UNPOLARIZED-NEUTRON BEAMS}

Neutron scattering experiments were performed using the NG-3 SANS instrument at the National Institute of Standards and Technology (NIST). The eight samples were stacked in a boronated aluminum sample holder and mounted inside a 9T horizontal-field cryomagnet with the [110] direction of each piece parallel to $\mathrm{B}$ and perpendicular to the neutron beam. The beam passed through a $0.8-\mathrm{cm}$ diameter hole in the holder parallel to the surface normal [110] of the stacked samples. We used three instrument configurations to measure a broad range of wavevector transfer $\vec{Q}$ (the difference between the incident and scattered wavevectors) at several fields. Images of the SANS using detector positions of $13.7 \mathrm{~m}$ with focusing neutron lenses and $5 \mathrm{~m}$ are shown in Figs. 4(a) and 4(b), respectively, for $B=+7 \mathrm{~T}$. The usual corrections have been made for detector efficiency, sample absorption, scattering from the empty sample holder and electronic noise, and the absolute units for the scattered intensity were determined in consideration of the total thickness $\sim 230 \mathrm{~nm}$ of the superlattice film (not including substrate). The SANS was also measured from eight uncoated sapphire substrates, and this was observed to be an order of magnitude weaker than the weakest scattering measured from the superlattice samples. We conclude therefore that the SANS is predominately from the superlattice film. Figures 4(c) and $4(\mathrm{~d})$ are corresponding images for $\mathrm{B}=-2 \mathrm{~T}$ after subtraction of the $+7 \mathrm{~T}$ data. Figure 4 shows anisotropic distributions of intensity on the detector, i.e., the SANS depends on $\phi$ (the angle between $\vec{Q}$ and $\vec{B}_{\mathrm{CF}}$ ). $\phi=0^{\circ}$ $\left(90^{\circ}\right)$ is the horizontal (vertical) direction on the SANS detector.

Using a detector distance of $12 \mathrm{~m}$, the SANS was measured in the range of $Q$ from 0.03 to $0.22 \mathrm{~nm}^{-1}$ for $900 \mathrm{~s}$ for each value of $\mathrm{B}$. The count rate integrated over the entire detector vs $\mathrm{B}$ for scattering within $20^{\circ}$ of $\phi=0^{\circ}$ and $\phi=90^{\circ}$ is shown in Fig. 5(a). The SANS is strongly dependent on B, being peaked about $\mathrm{B}_{\mathrm{EB}}$. For $\mathrm{B}>+2 \mathrm{~T}$, the SANS is relatively constant. Because according to our earlier $\mathrm{x}$-ray circular dichroism work, ${ }^{21}$ the $\mathrm{DyFe}_{2}$ magnetization is unresponsive to field [see Figure 1(a) in Ref. 21, the field dependence of the SANS can only come from the $\mathrm{YFe}_{2}$ layers.

SANS originates from differences of neutron-scattering length density across the lateral dimensions of the superlattice. ${ }^{29}$ The sources of these differences may be nuclear contrast, e.g., changes of mass density across low-angle grain boundaries, terraces, or magnetic contrast, e.g., from reorientation of magnetization from one magnetic domain to the next. The strong field dependence of the SANS [Fig. 5(a)] implies a magnetic source of the scattering. Further, the scattering of neutrons is sensitive to the component of the magnetization perpendicular to $\vec{Q} \cdot{ }^{30}$ To describe this, we use a Cartesian coordinate system with the $x$ axis defined along the applied field direction and the $z$ axis along the beam direction (out of the superlattice plane) [c.f. Fig. 4, inset]. For $\phi=0^{\circ}$, the scattering originates from the magnetization perpendicular to $\mathrm{B}$ (in the $y$ or $z$ directions), which is characteristic of $\sigma^{ \pm}$domains in the spin-chain model. For $\phi=90^{\circ}$ the scattering originates from the magnetization both out of the sample plane ( $z$ direction) or parallel to B ( $x$ direction) the latter is characteristic of $\sigma^{0}$ domains in the spin-chain model.

In Fig. 6 we show the SANS data integrated over (a) small and (b) large ranges of $Q$ as a function of $\phi$ for two fields $\mathrm{B}=+7$ (red) and $-2 \mathrm{~T}$ (black). The data exhibit field and $Q$ dependencies and anisotropy (i.e., variation with $\phi$ ). The solid curves were obtained by optimizing the parameters $\mathcal{N}^{2}+$ $\mathcal{M}_{z}^{2}, \mathcal{M}_{x}, \mathcal{M}_{y}$, and $C_{\mathcal{M}_{x} \mathcal{M}_{y}}$ in Eq. $(1)^{31}$ to minimize $\chi^{2}$-a goodness-of-fit metric. ${ }^{32}$ That is,

$$
\begin{aligned}
I^{\text {unpolarized }}= & 2\left(\mathcal{N}^{2}+\mathcal{M}_{z}^{2}\right)+2 \mathcal{M}_{x}^{2} \sin ^{2} \varphi+2 \mathcal{M}_{y}^{2} \cos ^{2} \varphi \\
& -2 C_{\mathcal{M}_{x} \mathcal{M}_{y}} \mathcal{M}_{x} \mathcal{M}_{y}|\sin 2 \varphi|
\end{aligned}
$$

$\mathcal{N}(\vec{Q})=\Sigma_{K} b_{K} e^{i \vec{Q} \cdot \vec{R}_{K}}$ is the Fourier transform of the nuclear scattering length $b$ for an ensemble of $K$-scattering objects with coordinates $\vec{R}_{K} \cdot \mathcal{M}_{J}(\vec{Q})=\Sigma_{K} p_{J, K} e^{i \vec{Q} \cdot \vec{R}_{K}}$ is the Fourier transform of the $J$ th Cartesian component of the magnetic scattering length $p . J=x$ corresponds to $\phi=0^{\circ}$, $J=y$ corresponds to $\phi=90^{\circ}$, and $J=z$ corresponds to the component parallel to the sample's surface normal. $p$ (units of $\mathrm{nm})$ is related to the volume magnetization by $M=\frac{u}{\mathrm{v}} \Sigma_{i} p_{i}$, where the summation is taken over the contents of the unit cell with volume $\mathrm{V}$ (units $\mathrm{cm}^{3}$ ) and $u=3.5 \times 10^{-15} \mathrm{emu} / \mathrm{nm}$.

We optimized Eq. (1) allowing only for positive values of $\mathcal{M}_{x}$ and $\mathcal{M}_{y} . C_{\mathcal{M}_{x} \mathcal{M}_{v}}$ is the correlation factor. $C_{\mathcal{M}_{x} \mathcal{M}_{v}}=1$ means that $\mathcal{M}_{x}$ and $\mathcal{M}_{y}$ are correlated. $C_{\mathcal{M}_{x} \mathcal{M}_{y}}=0$ means $\mathcal{M}_{x}$ and $\mathcal{M}_{y}$ are uncorrelated. $C_{\mathcal{M}_{x} \mathcal{M}_{v}}=-1$ means that $\mathcal{M}_{x}$ and $\mathcal{M}_{y}$ are correlated but with opposite signs (or opposing phases). Because $\vec{Q}$ contains an explicit dependence on $\phi, \mathcal{N}$ and $\mathcal{M}$ may exhibit dependence on both $|\vec{Q}|$ and $\phi$. Results from analysis of the unpolarized-beam SANS images are shown in Tables I and II for $\mathrm{B}=+7$ and $-2 \mathrm{~T}$, respectively. Generally the magnitudes of the components of $\mathcal{M}$ increase, particularly for small $Q$, as the exchange bias field is approached. 

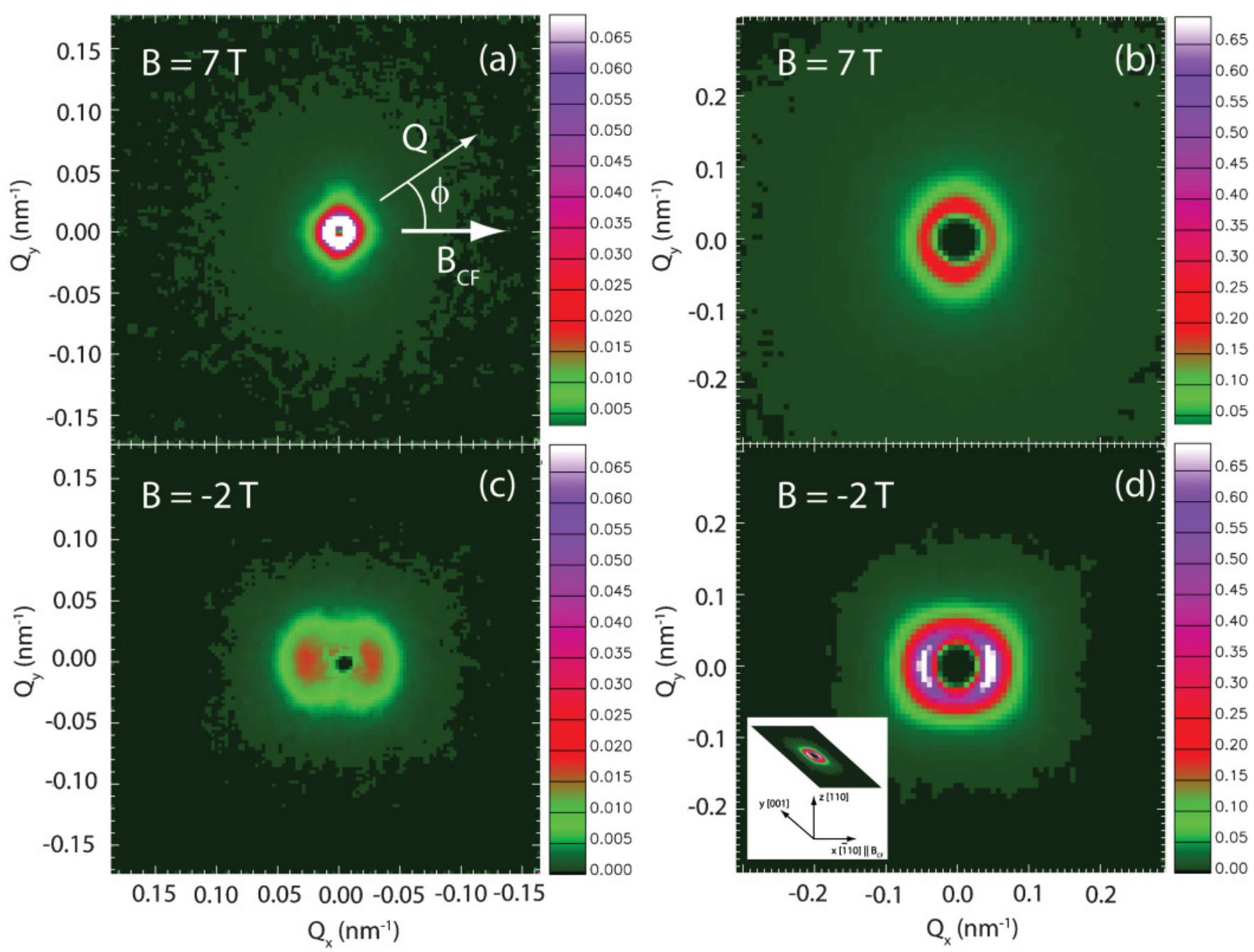

FIG. 4. (Color online) Neutron count rate (counts per second) per detector pixel recorded as a function of position across the detector for (a) and (b) $\mathrm{B}=+7 \mathrm{~T}$ at $12 \mathrm{~K}$ for different detector distances. Images (c) and (d) represent the intensity of data taken with $\mathrm{B}=-2 \mathrm{~T}$ after subtraction of the $+7 \mathrm{~T}$ data. Inset: Orientation of the sample axes with respect to the cooling field, which is parallel to the polarization axis of the neutron beam.

We were able to obtain excellent agreement with the data by treating $\mathcal{N}$ and the components of $\mathcal{M}$ as isotropic (scalar) parameters, i.e., $\mathcal{N}$ and $\mathcal{M}_{J}$ depended only upon $|\vec{Q}|$ and not on $\phi$. Thus, the anisotropy in the SANS images can be explained as a consequence of the dependence of neutron scattering on the component of magnetization perpendicular to $\vec{Q}^{30}$ giving rise to the trigonometric terms in Eq. (1).

Two equally good solutions are listed in the last column of Table II. The left solution is most consistent with the results of the polarized neutron-beam SANS experiment (discussed later).

Next, in order to better understand the $\phi$-dependencies of the data in Fig. 6, the SANS obtained over an extended region of $Q$ from detector distances of $5 \mathrm{~m}$ and $13.7 \mathrm{~m}$ for four combinations of B and $\phi$ is shown in Fig. 7. Three length scales are readily apparent in the data. An inflection at $Q \sim$ $0.1 \mathrm{~nm}^{-1}$ separates two length scales [identified as Features 1 and 2 in Fig. 7(a)], and an inflection at $Q \sim 0.02 \mathrm{~nm}^{-1}$ separates the remaining length scale [identified as Feature 3 in Fig. 7(b)].
The data were fitted to a combination of three squared Lorentzian functions,

$$
\begin{aligned}
I(Q)= & {\left[\frac{A_{1}}{\pi} \frac{\Gamma_{1}}{1+\left(\left(Q-Q_{1}\right) \Gamma_{1}\right)^{2}}\right]^{2}+\left[\frac{A_{2}}{\pi} \frac{\Gamma_{2}}{1+\left(Q \Gamma_{2}\right)^{2}}\right]^{2} } \\
& +\left[\frac{A_{3}}{\pi} \frac{\Gamma_{3}}{1+\left(Q \Gamma_{3}\right)^{2}}\right]^{2} .
\end{aligned}
$$

For appropriate values of $A_{i}, \Gamma_{i}$, and $Q_{1}$, Eq. (2) yields the black curves in Fig. 7. Except for $\Gamma_{3}$, the parameters were optimized independently for each measurement to minimize $\chi^{2}$. The individual-squared Lorentzian functions are shown by the colored curves. Squared Lorentzians, representing magnetic scattering from static magnetic correlations ${ }^{33-35}$ fit the data much better than Lorentzian functions, which have been used to represent fluctuations in a spin system, e.g., spin waves. ${ }^{36}$ The parameters obtained from fits to the $\phi=0^{\circ}$ and $90^{\circ}$ data are shown in Figs. 5(b) and 5(c).

Feature 1 (the blue curve, Fig. 7) was represented by a $Q$-shifted squared Lorentzian with parameters $A_{1}, \Gamma_{1}$, and 


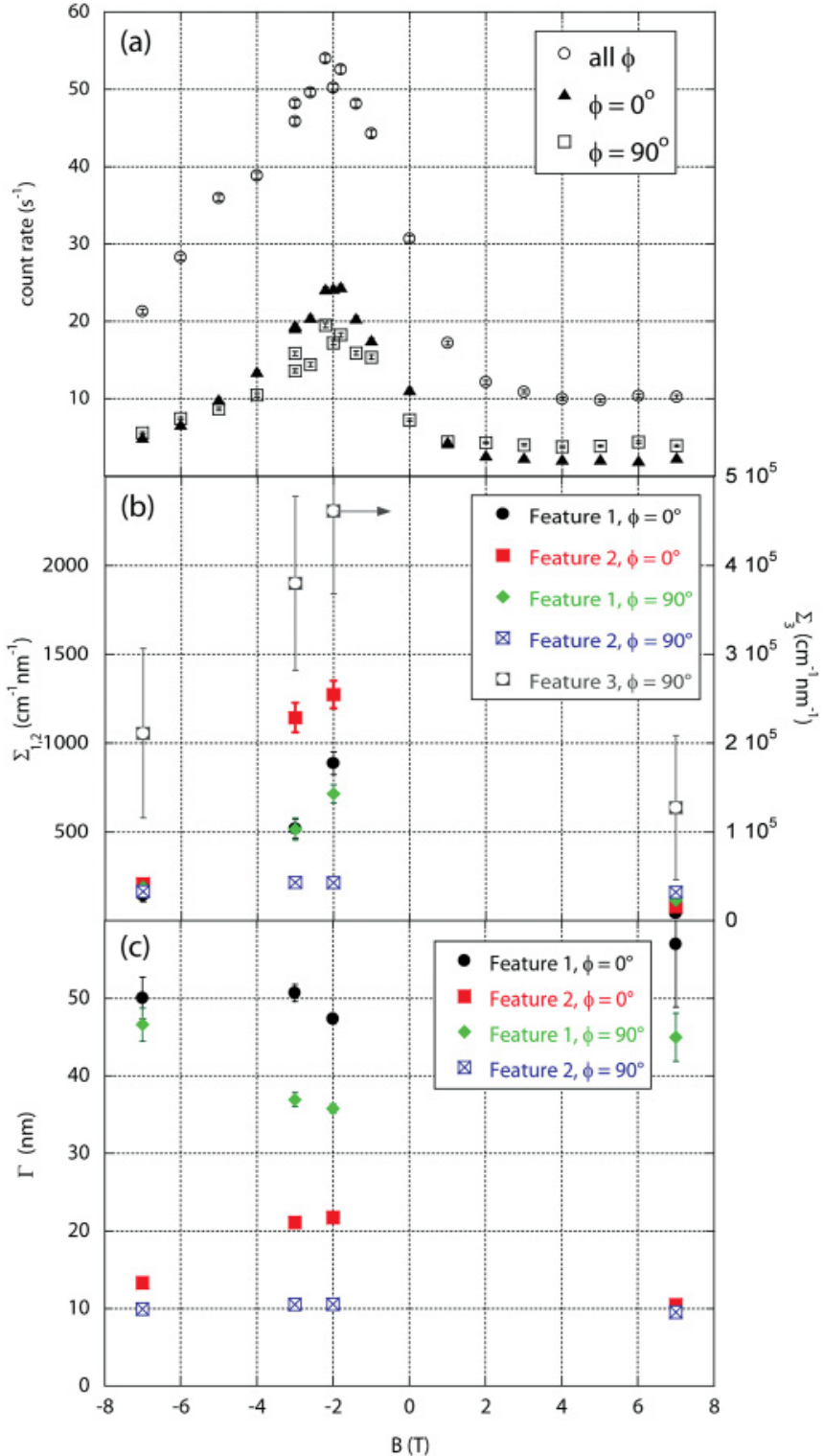

FIG. 5. (Color online) (a) SANS count rate integrated over the entire detector $(\circ)$ or within $20^{\circ}$ of $\phi=0^{\circ}(\boldsymbol{\Lambda})$ or $90^{\circ}(\square)$ vs B at $12 \mathrm{~K}$ after field cooling in $+1 \mathrm{~T}$. (b) Integrated intensities of Features 1, 2, and 3 and (c) characteristic dimensions of Features 1 and 2. (2-sigma errors shown.)

$Q_{1}$. The position $\left(Q_{1}\right)$ of Feature 1 was essentially constant: $(0.030 \pm 0.002) \mathrm{nm}^{-1}$ was measured for $\mathrm{B}=\mathrm{B}_{\mathrm{EB}}$ and $(0.036 \pm$ $0.002) \mathrm{nm}^{-1}$ for $|\mathrm{B}|=7 \mathrm{~T}$. The nonzero value of $Q_{1}$ implies a $\sim 190$-nm quasiperiodic variation of the magnetic structure that is the source of scattering (which later we attribute to the defects seen in the TEM picture, Fig. 2). The standard deviation of the periodicity $Q_{1}$ obtained for the different values of B was $8 \%$ of the mean. $\Gamma_{1}$ is related to the disorder in the periodic variation of the magnetic structure and is inversely proportional to the width intensity profile (blue curve, Fig. 7). $\Gamma_{1}=(46 \pm 6) \mathrm{nm}$ and depended somewhat upon B. $\Gamma_{1}$ tended to be narrower for $\phi=90^{\circ}$ than $\phi=0^{\circ}$ [Fig. 5(c)]. The integrated intensity of Feature 1, $\Sigma_{1}=\frac{A_{1}^{2} \Gamma_{1}}{2 \pi}$ [Fig. 5(b)], was strongly dependent upon B for all $\phi$. The moduli of the
TABLE I. Parameters of (1) fitted to the unpolarized beam SANS $(\mathrm{B}=+7 \mathrm{~T})$.

\begin{tabular}{lcc}
\hline \hline & $Q \in[0.03,0.05] \mathrm{nm}^{-1}$ & $Q \in[0.12,0.21] \mathrm{nm}^{-1}$ \\
\hline $\mathcal{N}^{2}+\mathcal{M}_{z}^{2}\left(\mathrm{~cm}^{-1}\right)$ & $0_{-0}^{+50}$ & $21 \pm 1$ \\
$\mathcal{M}_{x}^{2}\left(\mathrm{~cm}^{-1}\right)$ & $2116 \pm 188$ & $22 \pm 2$ \\
$\mathcal{M}_{y}^{2}\left(\mathrm{~cm}^{-1}\right)$ & $1089 \pm 136$ & $5 \pm 1$ \\
$C_{\mathcal{M}_{x} \mathcal{M}_{y}}$ & $0.7 \pm 0.1$ & $-0.5 \pm 0.1$ \\
\hline
\end{tabular}

components of $\mathcal{M}$ reported in the first columns of Tables I and II were obtained from integration of the SANS over a range of $Q$, where Feature 1 is significant. For large fields the components $\mathcal{M}_{x}$ and $\mathcal{M}_{y}$ corresponding to Feature 1 are well correlated. As the exchange bias field is approached, the strength of these components increases rapidly in an uncorrelated manner.

Feature 2 (red curve, Fig. 7) was well-represented by a squared Lorentzian profile centered at $Q=0$. Such a profile can result from domains that are randomly distributed across the lateral dimensions of the sample. $\Gamma_{2}$ is the characteristic size of the scattering source for Feature 2 (which later we attribute to the widths of domain walls for example around the islands seen in Fig. 2). For $\phi=90^{\circ}$ the characteristic size was $(10 \pm 1) \mathrm{nm}$ for all $\mathrm{B}$. However, for $\phi=0^{\circ}$ this size ranged between $10 \mathrm{~nm}$ for large $|\mathrm{B}|$ and $21 \mathrm{~nm}$ for $\mathrm{B}=\mathrm{B}_{\mathrm{EB}}$. Likewise, the integrated intensity of Feature 2, $\Sigma_{2}=\frac{A_{2}^{2} \Gamma_{2}}{2 \pi}$ [Fig. 5(b)], was independent of $\mathrm{B}$ for $\phi=90^{\circ}$ and strongly dependent upon $\mathrm{B}$ for $\phi=0^{\circ}$ (i.e., for magnetization perpendicular to B). Features 1 and 2 contribute to the intensity in Fig. 5(a). The moduli of the components of $\mathcal{M}$ reported in the second columns of Tables I and II were obtained from integration of the SANS over a range of $Q$ where Feature 2 is dominant. For large fields the components $\mathcal{M}_{x}$ and $\mathcal{M}_{y}$ corresponding to Feature 2 are weak and grow in strength as the exchange bias field is approached. These components are partially correlated for all fields, though with opposite signs.

Finally, Feature 3 (green curve, Fig. 7) represents the SANS at $\phi=90^{\circ}$ that diverges as $Q$ approaches zero. Since we lack data for small $Q$ necessary to resolve length scales greater than $\sim 628 \mathrm{~nm}$, we fixed $\Gamma_{3}=628 \mathrm{~nm}$ and fitted only the amplitude parameter for this contribution, i.e., $A_{3}$. Owing to the large value of $\Gamma_{3}$, the third term in (2) is essentially equal to $\left(\frac{A_{3}}{\pi \Gamma_{3}}\right) Q^{-4}$, which is characteristic of Porod scattering from surfaces or interfaces, e.g., magnetic domain walls, extending over length scales much greater than $600 \mathrm{~nm}$ (which later we attribute to $\sigma^{0}$ domains). Feature 3 does not contribute to the

TABLE II. Parameters of (1) fitted to the unpolarized beam SANS $(\mathrm{B}=-2 \mathrm{~T})$.

\begin{tabular}{lcc}
\hline \hline & $Q \in[0.03,0.05] \mathrm{nm}^{-1}$ & $Q \in[0.12,0.21] \mathrm{nm}^{-1}$ \\
\hline $\mathcal{N}^{2}+\mathcal{M}_{z}^{2}\left(\mathrm{~cm}^{-1}\right)$ & $1824 \pm 196$ & $31 \pm 3\left(0_{-1}^{+2}\right)$ \\
$\mathcal{M}_{x}^{2}\left(\mathrm{~cm}^{-1}\right)$ & $4900 \pm 284$ & $34 \pm 3(66 \pm 3)$ \\
$\mathcal{M}_{y}^{2}\left(\mathrm{~cm}^{-1}\right)$ & $11236 \pm 428$ & $22 \pm 3(53 \pm 3)$ \\
$C_{\mathcal{M}_{x} \mathcal{M}_{y}}$ & $0.1 \pm 0.1$ & $-1_{-0}^{+0.2}(-0.5 \pm 0.1)$ \\
\hline \hline
\end{tabular}



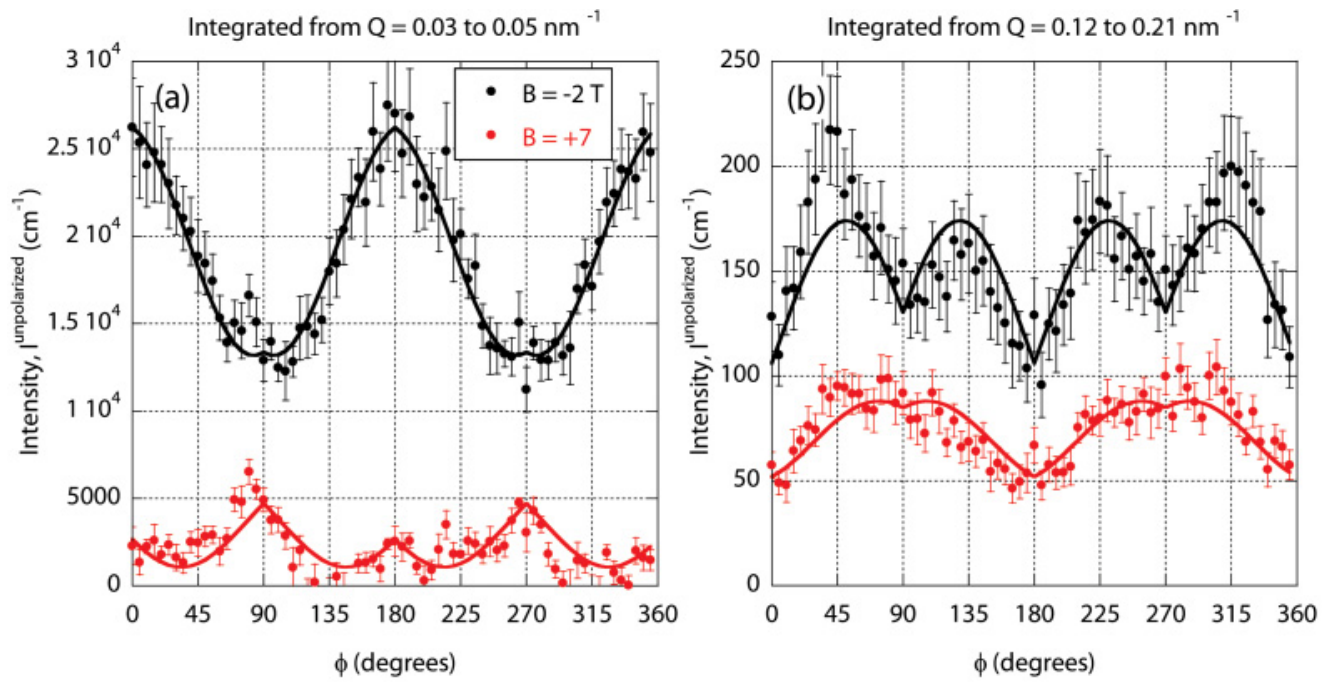

FIG. 6. (Color online) The SANS intensity integrated with an annulus (a) close to and (b) far from the origin. The integrated intensity is plotted as a function of angle $\phi$ from the applied field. Solid curves were obtained from (1) and parameters are listed in Tables I and II. (1-sigma errors shown.)
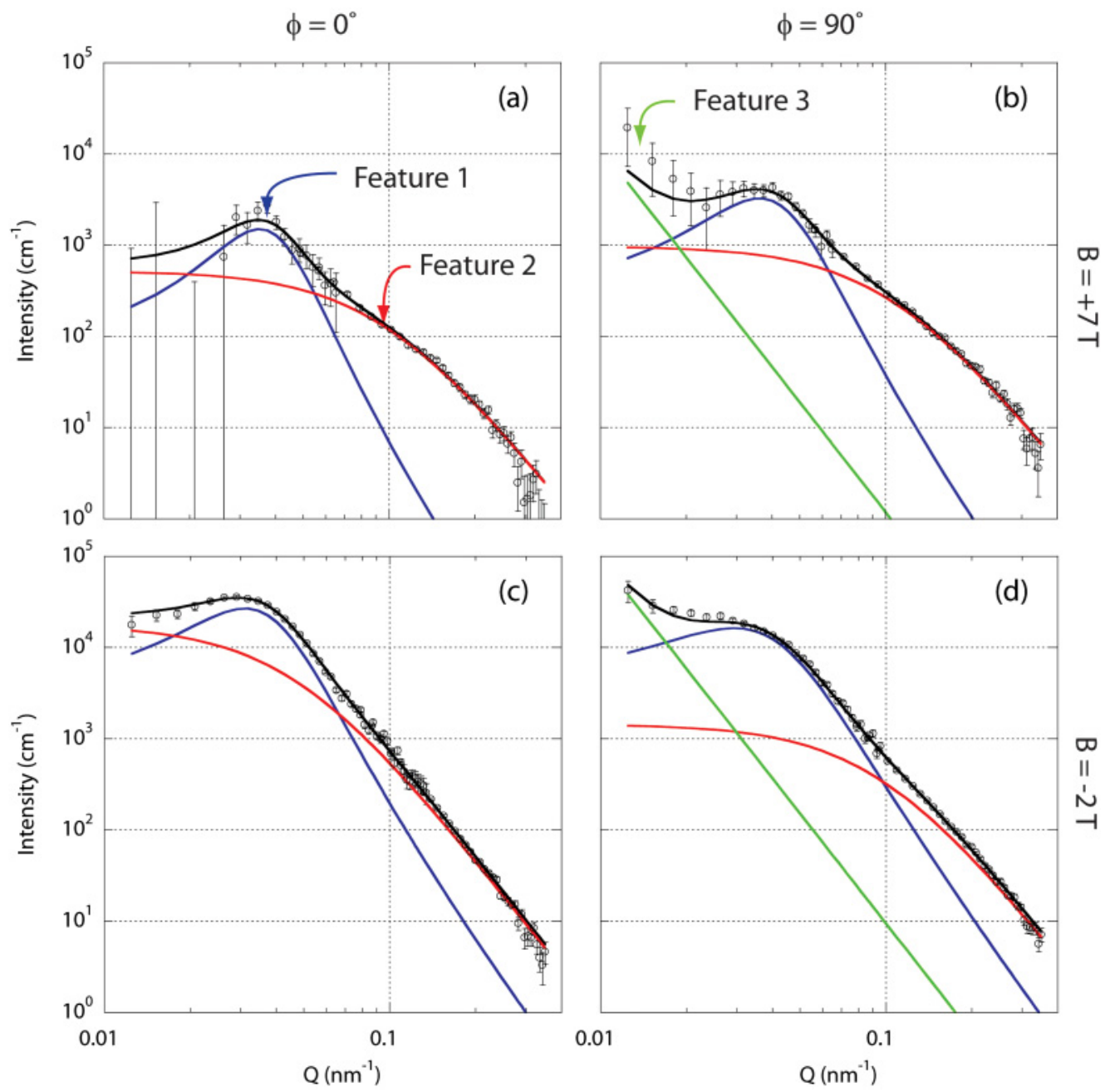

FIG. 7. (Color online) Intensity plotted for $\mathrm{B}=+7 \mathrm{~T}$ (a) and (b) and $-2 \mathrm{~T}$ (c) and (d) as a function of $Q$ for SANS integrated within $\pm 20^{\circ}$ of $\phi=0^{\circ}$ (a) and (c) and $90^{\circ}$ (b) and (d). The curves were obtained from squared Lorentzian functions (see Eq. (2)) fitted to the data. (1-sigma errors shown.) 

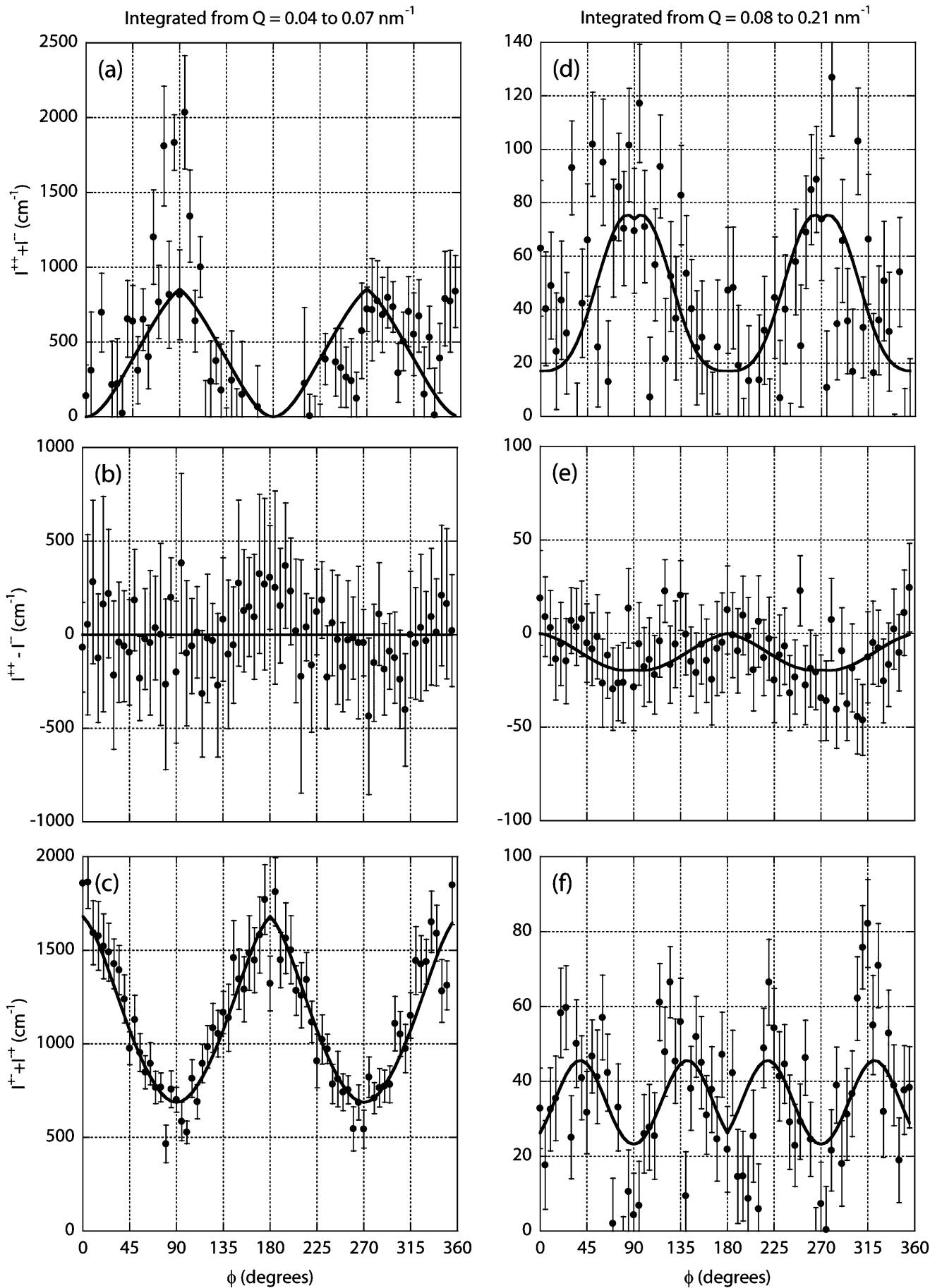

FIG. 8. Intensities integrated with an annulus (a) close to and (b) far from the origin. The integrated intensity is plotted as a function of angle from $\phi$ from the applied field for (a) and (d) the sum of the NSF SANS, (b) and (e) the difference of the NSF SANS and (c) and (f) the sum on the SF SANS for $12 \mathrm{~K}$ and $\mathrm{B}=-1.2 \mathrm{~T}$. Solid curves obtained from Eqs. (3)-(5) and parameters listed in Table 4. (1-sigma errors shown.)

intensity shown in Fig. 5(a) since the scattering associated with Feature 3 lies within the beam stop for the intermediate detector setting used to record these data. Feature $3\left(\phi=90^{\circ}\right)$ is strongly dependent upon $\mathrm{B}$ with a maximum at $\mathrm{B}=\mathrm{B}_{\mathrm{EB}}$. Note that the squared Lorentzian used to fit Feature 2 diverges as $Q$ becomes small, thus, a portion of Feature 2 for $\phi=90^{\circ}$ may contribute to Feature 3.

\section{SANS WITH POLARIZED-NEUTRON BEAMS}

In order to facilitate direct separation of the nuclear scattering from that of the magnetization components, SANS measurements were carried out on the NG-3 SANS instrument at the NIST using a polarized-neutron beam and a ${ }^{3} \mathrm{He}-$ polarization filter to analyze the SANS. ${ }^{37}$ The eight samples were placed in a cryostat between the pole pieces of an 
electromagnet and cooled in a $+1 \mathrm{~T}$ field to $12 \mathrm{~K}$. The polarization axis of the neutron beam was parallel to the $x$-direction, [110], and B. For each of two fields $+1 \mathrm{~T}$ and $-1.2 \mathrm{~T}$, four polarized neutron-scattering cross sections, the two nonspin-flip (NSF) intensities, $I^{++}$and $I^{--}$, and the two spin-flip (SF) intensities, $I^{+-}$and $I^{-+}$, were measured representing all combinations of incident and scattered-beam polarization. The subscript $+(-)$ denotes neutron polarization parallel (opposite) to the $x$-direction. The data have been corrected for efficiencies of the polarizer and analyzer. ${ }^{38}$

Integration of the SANS over ranges of $Q$ close to and far from the origin are shown in Figs. 8(a)-8(f), respectively. These integrations show strong dependencies on $Q$ and $\phi$. The solid curves were obtained by simultaneously optimizing scalar values for the parameters $\mathcal{N}^{2}, \mathcal{M}_{z}^{2}, \mathcal{M}_{x}, \mathcal{M}_{y}$, $C_{\mathcal{M}_{x} \mathcal{M}_{y}}, C_{\mathcal{N M}_{x}}$, and $C_{\mathcal{N M}_{y}}$ (see Table III) in Eqs. (3)-(5) (based upon a generalization of equations in Ref. 31) to achieve a best fit to the data shown in Fig. 8,

$$
\begin{aligned}
& I^{++}+I^{--}=2 \mathcal{N}^{2}+2 \mathcal{M}_{x}^{2} \sin ^{4} \varphi+2 \mathcal{M}_{y}^{2} \sin ^{2} \varphi \cos ^{2} \varphi \\
& -2 C_{\mathcal{M}_{x} \mathcal{M}_{y}} \mathcal{M}_{x} \mathcal{M}_{y} \sin ^{2} \varphi|\sin 2 \varphi| \\
& I^{++}-I^{--}=4 C_{\mathcal{N} \mathcal{M}_{x}} \mathcal{N} \mathcal{M}_{x} \sin ^{2} \varphi-2 C_{\mathcal{N} \mathcal{M}_{y}} \mathcal{N} \mathcal{M}_{y}|\sin 2 \varphi| \\
& I^{+-}+I^{-+}=2 \mathcal{M}_{z}^{2}+2 \mathcal{M}_{x}^{2} \sin ^{2} \varphi \cos ^{2} \varphi+2 \mathcal{M}_{y}^{2} \cos ^{4} \varphi \\
& -2 C_{\mathcal{M}_{x} \mathcal{M}_{y}} \mathcal{M}_{x} \mathcal{M}_{y} \cos ^{2} \varphi|\sin 2 \varphi| \text {. }
\end{aligned}
$$

Given the good fit, the data are consistent with the assumption that $\mathcal{N}(Q)$ and the components of $\mathcal{M}(Q)$ are spatially isotropic, i.e., the components do not depend upon $\phi$. Thus, the intensities of the spin-dependent cross sections are straightforwardly related to $\mathcal{N}(Q)$ and $\mathcal{M}(Q)$ by ${ }^{31}$

$$
\begin{gathered}
I_{\varphi=0}^{++}=I_{\varphi=0}^{-}=\mathcal{N}^{2} \\
I_{\varphi=0}^{+-}=I_{\varphi=0}^{-+}=\mathcal{M}_{z}^{2}+\mathcal{M}_{y}^{2} \\
I_{\varphi=90}^{ \pm \pm}=\mathcal{N}^{2}+\mathcal{M}_{x}^{2} \pm 2 C_{\mathcal{N} \mathcal{M}_{x}} \mathcal{N} \mathcal{M}_{x} \\
I_{\varphi=90}^{+-}=I_{\varphi=90}^{-+}=\mathcal{M}_{z}^{2} .
\end{gathered}
$$

These equations allow us to isolate the $Q$-dependencies of $\mathcal{N}^{2}, \mathcal{M}_{z}^{2}, \mathcal{M}_{y}^{2}$, and $\mathcal{M}_{x}^{2}$ (Fig. 9) from the polarizationdependent SANS measurements. The $Q$-dependence of the structural scattering is very nearly $Q^{-2}$ (i.e., consistent with a Lorentzian) [solid line Fig. 9(a)]. For $Q>0.04 \mathrm{~nm}^{-1}$ neither the structural scattering nor $\mathcal{M}_{x}^{2}$ exhibit field dependence in

TABLE III. Table 3. Parameters of Eqs. (3)-(5) fitted to the polarized beam SANS $(\mathrm{B}=-1.2 \mathrm{~T})$.

\begin{tabular}{lcc}
\hline \hline & $Q \in[0.04,0.07] \mathrm{nm}^{-1}$ & $Q \in[0.08,0.21] \mathrm{nm}^{-1}$ \\
\hline $\mathcal{N}^{2}\left(\mathrm{~cm}^{-1}\right)$ & $0 \pm 9$ & $9 \pm 2$ \\
$\mathcal{M}_{z}^{2}\left(\mathrm{~cm}^{-1}\right)$ & $341 \pm 9$ & $12 \pm 1$ \\
$\mathcal{M}_{x}^{2}\left(\mathrm{~cm}^{-1}\right)$ & $429 \pm 49$ & $28 \pm 4$ \\
$\mathcal{M}_{y}^{2}\left(\mathrm{~cm}^{-1}\right)$ & $497 \pm 39$ & $2 \pm 1$ \\
$C_{\mathcal{M}_{x} \mathcal{M}_{y}}$ & $0.16 \pm 0.07$ & $-1.0_{-0}^{+0.5}$ \\
$C_{\mathcal{N} \mathcal{M}_{x}}$ & $0 \pm 1$ & $-0.3 \pm 0.2$ \\
$C_{\mathcal{N} \mathcal{M}_{y}}$ & $0 \pm 1$ & $0.4_{-1}^{+0.6}$ \\
\hline \hline
\end{tabular}

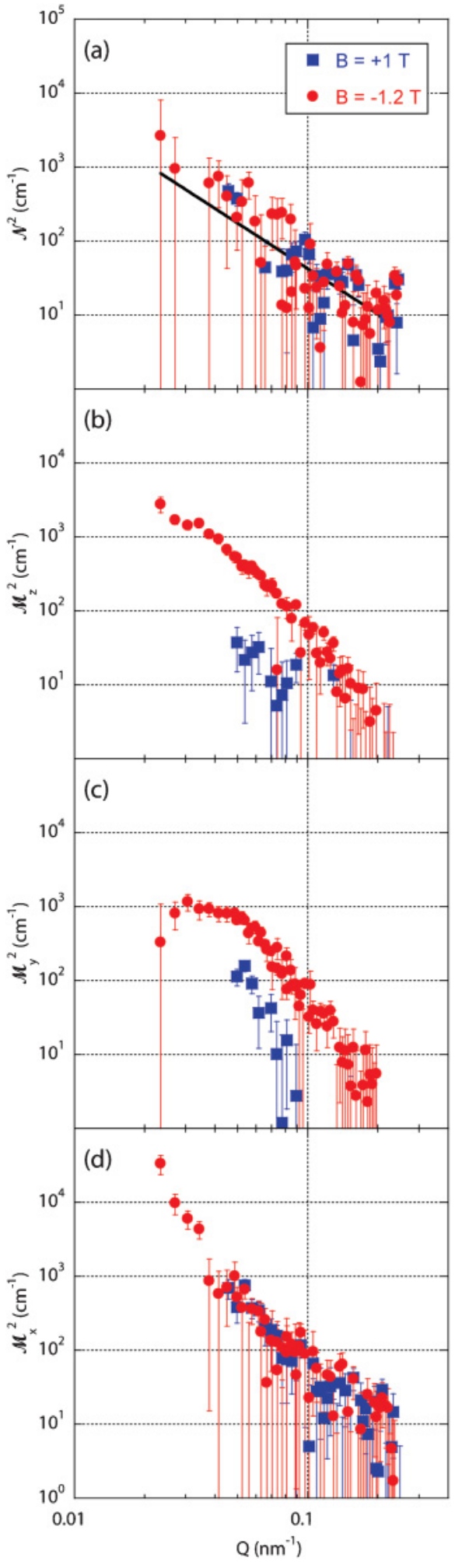

FIG. 9. (Color online) Variation of (a) the structural scattering $\mathcal{N}^{2}$ and (b)-(d) components of the magnetic scattering $M^{2}$ for B $=+1 \mathrm{~T}$ and $-1.2 \mathrm{~T}$ at $12 \mathrm{~K}$ obtained from application of Eqs. (6) through (9) to $I^{ \pm \pm}(Q)$ and $I^{ \pm \mp}(Q)$. (1-sigma errors shown.)

the range of $\mathrm{B}$ from +1 to $-1.2 \mathrm{~T}$. However, the components $\mathcal{M}_{z}^{2}$ and $\mathcal{M}_{y}^{2}$ are strongly field dependent. 


\section{DISCUSSION}

The SANS experiments have identified several key insights. The first insight is that $\mathcal{M}(Q)$ is quasiperiodic corresponding to length scales of $\sim 190 \mathrm{~nm}$ in the sample plane (Feature 1). The polarized-beam SANS experiment provided evidence that Feature 1 arises from a combination of in- and out-of-plane components of $\mathcal{M}$. From the unpolarized-beam SANS experiment, as B reaches the exchange bias field, $\mathcal{M}_{x}^{2}$, $\mathcal{M}_{y}^{2}$, and $\mathcal{M}_{z}^{2}$ collectively increase. These components appear to act in concert over the entire $Q$-range (i.e., for Features 1 and 2 ) thus suggesting rotation of magnetization during the reversal process. Reversal of magnetization leading to an increase of $\mathcal{M}_{y}^{2}$ and $\mathcal{M}_{z}^{2}$ is consistent with $\sigma^{ \pm}$domains, while an increase of $\mathcal{M}_{x}^{2}$ is consistent with $\sigma^{0}$ domains.

Though their relative magnitudes differ as a function of field, there is no evidence from either unpolarized-beam or polarized-beam experiments to suggest that $\mathcal{M}_{x}, \mathcal{M}_{y}$, and $\mathcal{M}_{z}$ vary as a function of $\phi$. Given the strong unidirectional magnetic anisotropy of our system, the observation that $\mathcal{M}$ (the Fourier transform of $M$ ) does not depend upon $\phi$ is surprising. One could speculate that since SANS images of magnetism inherently depend upon $\phi$ (due to the dependence of SANS on the Halpern-Q-vector ${ }^{30}$ ), the technique may be limited in its ability to discern subtle deviations of scattering arising from magnetic anisotropy. However, "cloverleaf" patterns in SANS images have been observed by others ${ }^{24}$ in different systems and attributed to the influence of a magnetostatic dipolar stray field outside magnetic domains that deflects spins inside the domains. We suggest that while our system has considerable magnetic anisotropy, our data are consistent with a reversal process that includes the formation of an isotropic distribution of nm-sized domains and domain walls.

The second insight is that the magnetic scattering is not fully suppressed at $\mathrm{B}= \pm|7| \mathrm{T}$, since Features 1,2 , and 3 persist to the highest fields. This insight is further confirmed by the lack of field dependence of $\mathcal{M}_{x}^{2}$ for $Q>0.04 \mathrm{~nm}^{-1}$ [Fig. 9(d)] in the polarized-beam data as the field is reduced from $+1 \mathrm{~T}$ to $-1.2 \mathrm{~T}$. As the field is further reduced to $-2 \mathrm{~T}, \mathcal{M}_{x}^{2}$ does increase (see Tables I and II). This behavior is expected for $\sigma^{0}$ domains. Specifically, the magnetizations of $\sigma^{0}$ domains do not reverse until the exchange bias field is reached, unlike the case for the $\sigma^{ \pm}$domains, which begin reversal in small positive fields. $\mathcal{M}_{x}^{2}$ is nevertheless nonzero at $\mathrm{B}=+7 \mathrm{~T}$.

The third insight - the significance of the structural scattering and its relationship to magnetic scattering-leads to possible identification for an origin of the pinned interfacial magnetization. The analysis of the $\phi$-dependence of the polarized-beam data suggests $\mathcal{N}$ and $\mathcal{M}_{x}$ may be weakly correlated for large $Q$ (corresponding to Feature 2). Defects in the film (Fig. 2) with length scale comparable to those inferred from SANS may be responsible for the pinned magnetic interfaces in $\mathrm{DyFe}_{2}$. The fact that the correlation between $\mathcal{N}$ and $\mathcal{M}_{x}$ is not strong (see $C_{\mathcal{N} \mathcal{M}_{x}}$, Table III), and zero for small $Q$, can be partly attributed to the difference in the $Q$-dependencies of the structural scattering (which varies as $Q^{-2}$ ) and the magnetic scattering (which varies between $Q^{-3}$ and $\left.Q^{-4}\right)$. Also, the defects do not extend throughout the entire film, thus, the structural and magnetic SANS may be averaging over different portions of the sample.
We propose a qualitative model for magnetic inhomogeneity that gives rise to the SANS observations. The "Plum Pudding Model" (Fig. 10) is general by design but accounts for the contrasting field dependencies of the three magnetic correlation lengths [Fig. 5(c)]. Starting at the largest length scales, e.g., microns, the model consists of domains with the bulk of their magnetizations equally aligned parallel or opposite to $\mathrm{B}$ at $\mathrm{B}_{\mathrm{EB}}$. These domains were identified as $\sigma^{0}$ domains in Ref. 21. The existence of these domains is implied by magnetometry and polarized-neutron reflectometry data. However, Feature 3 is also consistent with a variation of $\mathcal{M}_{x}$ along [001] with 600+ nm length scale [Figs. 7(b) and $7(\mathrm{~d})]$. The SANS data are not sensitive to a variation of $\mathcal{M}_{x}$ along [110]. ${ }^{30}$ The micron-size domains are shown by gray and blue in Fig. 10. These regions constitute the pudding.

The "Plums" (shown as circular regions in Fig. 10, bottom) are a source of nm-length-scale nonuniform magnetization giving rise to SANS. We associate the "Plums" with the structural defects or islands identified in Fig. 2 since the length scales are comparable. The magnetization in the "Plums" is canted away from the applied field in the $y$-and $z$-directions (given the large anisotropy of $\mathrm{DyFe}_{2}$ and the bending of the $\mathrm{DyFe}_{2}$ and $\mathrm{YFe}_{2}$ layers about the islands, these defects would naturally lead to some canted magnetization). Plums within groups (shown by dashed ellipses, Fig. 10) are spaced roughly $\sim 190 \mathrm{~nm}$ apart, and the magnetizations of plums in a group are similarly canted. The sizes of the "Plums" are undetermined (though they must be smaller than their $\sim 190 \mathrm{~nm}$ separation). Correlation and variation of the spacing give rise to the position and width of Feature 1. For $\mathrm{B}=\mathrm{B}_{\mathrm{EB}}$ the magnetization of the "Plums" is most canted from B. As $\left|\mathrm{B}-\mathrm{B}_{\mathrm{EB}}\right|$ increases, the canting decreases; consequently, the SANS decreases (see bottom left and right panels, Fig. 10). Furthermore, for large $\mathrm{IB}-\mathrm{B}_{\mathrm{EB}} \mid$ the density of "Plums" may also decrease (for example, see bottom left panel, Fig. 10). In this model, 20-nm-wide domain walls in the "Pudding" and between "Plums" and "Pudding" give rise to Feature 2. Again as $\mid \mathrm{B}-\mathrm{B}_{\mathrm{EB}}$ l increases, the number of domain walls decreases, thus, also contributing to the observed field dependence of the intensity of Feature 2. Attributes of the Plum Pudding Model include rotation of magnetization away from [110] akin to $\sigma^{ \pm}$ domains.

One may ask why does the domain magnetization deviate at all from the applied field? An explanation is that the magnetic-anisotropy axis of the $\mathrm{YFe}_{2}$ may not be parallel to [110]. Evidence that this is the case is seen by the depressed remanent magnetization. Alternatively, the $\mathrm{YFe}_{2}$ layers may not be parallel to the sample's surface (indeed some layers are not parallel, see Fig. 2). One insight of the 1D-spin-chain model reported in Ref. 21 was that in order to realize the $\sigma^{ \pm}$ mechanism, the easy axis of $\mathrm{YFe}_{2}$ was parallel to [001] and significantly different than that of $\mathrm{DyFe}_{2}$ (which is parallel to [110]). A superlattice with still larger exchange bias and/or better attributes for a permanent magnet could be realized if the remanent magnetization of the superlattice could be made larger. Our study suggests that key to realizing this improvement is to suppress the rotatable $\sigma^{ \pm}$mechanism by tailoring extrinsic defects so as to avoid nucleation of $\sigma^{ \pm}$domains by suppressing canted magnetization and by 


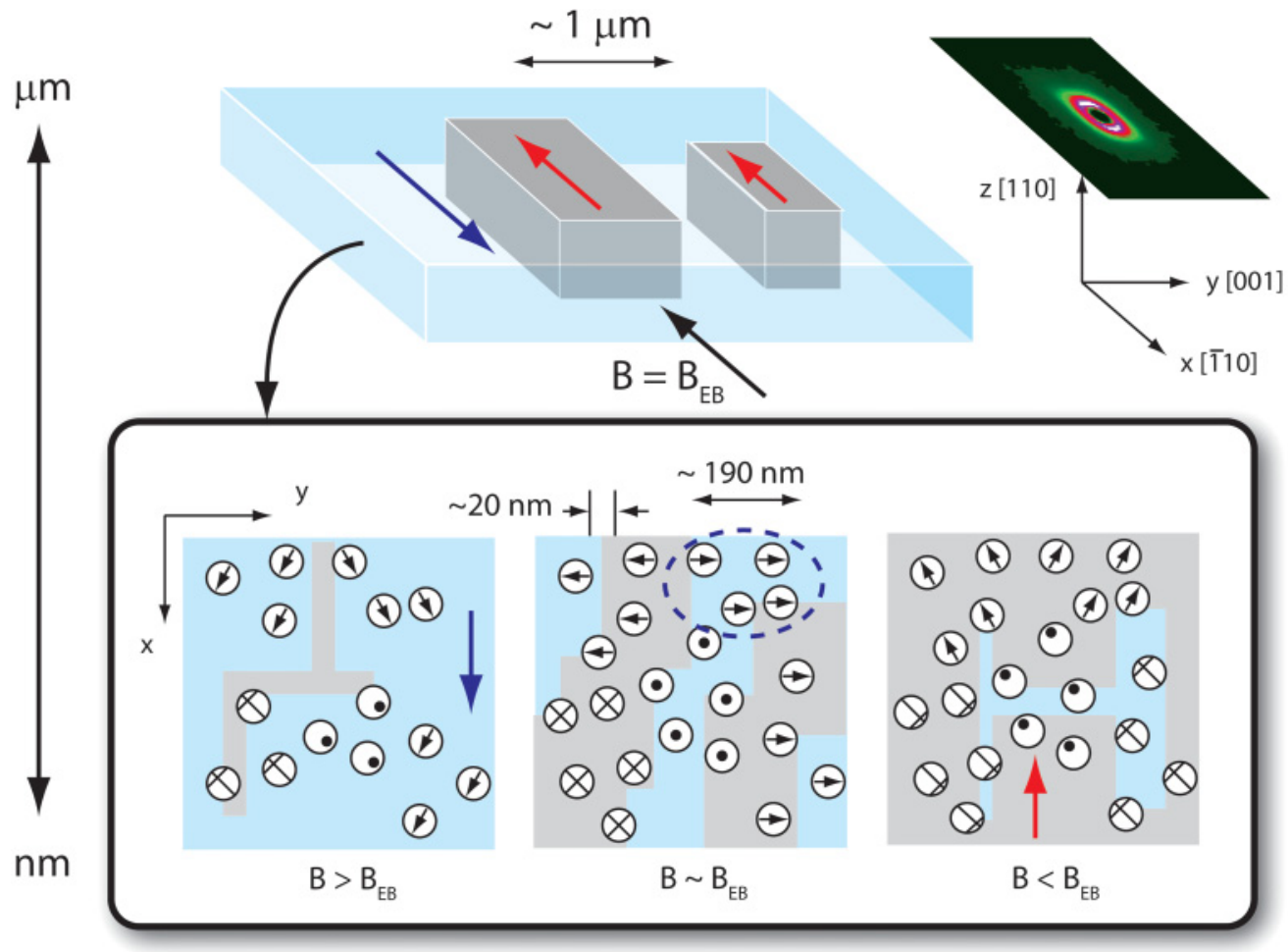

FIG. 10. (Color online) Model of the inhomogeneous magnetization state near $\mathrm{B}_{\mathrm{EB}}$ motivated by the SANS study. The component of $\mathcal{M}$ along B is nonuniform at micron-length scales parallel to [001] and greater than the micron-length scale parallel to [1110]. Gray (blue) regions are ones with magnetization parallel (opposite) to $\mathrm{B}_{\mathrm{EB}}$. Domain walls occur at interfaces between gray and blue regions and interfaces around the nm-sized domains depicted with circular shapes.

engineering the anisotropy of the soft layer magnetization to be parallel to that of the pinned magnetization.

\section{CONCLUSIONS}

In our Laves phase superlattice, we found two sources of field-dependent magnetic scattering correlated with the exchange bias-both affecting the magnetization of $\mathrm{YFe}_{2}$ on nm length scales. Remarkably, the first source is somewhat periodically ordered with a period of $\sim 190 \mathrm{~nm}$. The second consists of magnetic domains or domain walls as small as $10 \mathrm{~s}$ of $\mathrm{nm}$. Using polarized-neutron beams with polarization analysis of the SANS, we further isolated the magnetic scattering from the structural scattering, separated the magnetic scattering into orthogonal components, and show that magnetization reversal at the bias field occurs via rotation of the magnetization in and out of the sample plane. We provided a model showing how the magnetization reversal may occur and offered a solution to inhibit its reversal that should be applicable to many other systems.

Strong magnetic scattering was accompanied with strong structural (nuclear) scattering. While the SANS images are anisotropic, the underlying sources of structural and magnetic scatterings depend only upon the magnitude of the wavevector transfer $|Q|$ and not its direction. The structural and magnetic scatterings are certainly not correlated for all fields; nevertheless, extrinsic structural features of the sample, e.g., islands, terraces, etc., may influence nucleation of magnetic domains that have a comparable length scale. These periodic domains subsequently grow or shrink in response to field.

The pronounced enhancement of the integrated intensity of the SANS at $\mathrm{B}_{\mathrm{EB}}$, suggests a strong correlation between exchange bias and the preponderance of $<200-n m$-sized domains in the FM (i.e., $\mathrm{YFe}_{2}$ ). In other systems a positive correlation between large exchange bias and a preponderance of structural defects has been observed. ${ }^{19}$ An explanation for the very large exchange bias in the $\mathrm{DyFe}_{2} / \mathrm{YFe}_{2}$ system may be that small ferromagnetic domains average the anisotropy of the pinned magnetic layer (the $\mathrm{DyFe}_{2}$ ) over small regions, which at the nm-length scale appear uniform. Uniformity of interface coupling between a FM and the surface of uncompensated pinned magnetization (as realized in the $\mathrm{DyFe}_{2}$ FI) should promote large exchange bias. This reasoning may explain why the $\mathrm{DyFe}_{2} / \mathrm{YFe}_{2}$ system is a system that exhibits among the largest exchange bias observed to date.

\section{ACKNOWLEDGMENTS}

This work was supported by the Office of Basic Energy Science, US Department of Energy, BES-DMS funded by the Department of Energy's Office of Basic Energy Science. Los Alamos National Laboratory is operated by Los Alamos National Security LLC under DOE Contract DE-AC5206NA25396. This work utilized facilities at the NIST Center for Neutron Research supported in part by the National Science Foundation under Agreement No. DMR-0454672. M.R.F. gratefully acknowledges financial assistance from the Université Nancy. M.L. acknowledges support from DanScatt. 
*Deceased

${ }^{\dagger}$ fitz@lanl.gov

${ }^{1}$ E. F. Kneller and R. Hawig, IEEE Trans. Magn. 27, 3588 (1991).

${ }^{2}$ W. H. Meiklejohn and C. P. Bean, Phys. Rev. 102, 1413 (1957).

${ }^{3}$ W. H. Meiklejohn and C. P. Bean, Phys. Rev. 105, 904 (1957).

${ }^{4}$ J. Nogués and I. K. Schuller, J. Magn. Magn. Mater. 192, 203 (1999).

${ }^{5}$ A. E. Berkowitz and K. Takano, J. Magn. Magn. Mater. 200, 552 (1999).

${ }^{6}$ R. L. Stamps, J. of Phys. D: Appl. Phys. 33, R247 (2000).

${ }^{7}$ M. Kiwi, J. Magn. Magn. Mater. 234, 584 (2001).

${ }^{8}$ F. Radu and H. Zabel, Exchange Bias Effect of FerroAntiferromagnetic Heterostructures, Springer Tracts in Modern Physics (Berlin, Springer-Verlag) 227, pp. 97-184 (2008).

${ }^{9}$ M. R. Fitzsimmons, D. Lederman, M. Cheon, H. Shi, J. Olamit, I. V. Roshchin, and I. K. Schuller, Phys. Rev. B 77, 224406 (2008).

${ }^{10}$ H. Ohldag, H. Shi, E. Arenholz, J. Stohr, and D. Lederman, Phys. Rev. Lett. 96, 027203 (2006).

${ }^{11}$ M. Cheon, Z. Y. Liu, and D. Lederman, J. Appl. Phys. 101, 09E503 (2007).

${ }^{12}$ Z. P. Li, C. W. Miller, I. V. Roshchin, and I. K. Schuller, Phys. Rev. B 76, 014423 (2007).

${ }^{13}$ I. V. Roshchin, O. Petracic, R. Morales, Z. P. Li, X. Batlle, and I. K. Schuller, Euro. Phys. Lett. 71, 297 (2005).

${ }^{14}$ C. Binek, S. Polisetty, X. He, and A. Berger, Phys. Rev. Lett. 96, 067201 (2006).

${ }^{15}$ A. E. Berkowitz, J. I. Hong, S. K. McCall, E. Shipton, K. T. Chan, T. Leo, and D. J. Smith, Phys. Rev. B 81, 134404 (2010).

${ }^{16}$ I. Schmid, M. A. Marioni, P. Kappenberger, S. Romer, M. ParlinskaWojtan, H. J. Hug, O. Hellwig, M. J. Carey, and E. E. Fullerton, Phys. Rev. Lett. 105, 197201 (2010).

${ }^{17}$ O. Hellwig, A. Berger, and E. E. Fullerton, J. Magn. Magn. Mater. 290, 1 (2005).

${ }^{18}$ U. Nowak, K. D. Usadel, J. Keller, P. Miltenyi, B. Beschoten, and G. Guntherodt, Phys. Rev. B 66, 014430 (2002).

${ }^{19}$ M. R. Ghadimi, B. Beschoten, and G. Güntherodt, Appl. Phys. Lett. 87, 261903 (2005).

${ }^{20}$ B. Dieny, J. Magn. Magn. Mater. 136, 335 (1994).

${ }^{21}$ M. R. Fitzsimmons, C. Dufour, K. Dumesnil, J. Dou, and M. Pechan, Phys. Rev. B 79, 144425 (2009).
${ }^{22}$ Y. Henry, S. Mangin, and F. Montaigne, Phys. Rev. B 69, 140401(R) (2004).

${ }^{23}$ J. McCord, Y. Henry, T. Hauet, F. Montaigne, E. E. Fullerton, and S. Mangin, Phys. Rev. B 78, 094417 (2008).

${ }^{24}$ A. Michels and J. Weissmüller, Rep. Prog. Phys. 71, 066501 (2008).

${ }^{25}$ M. R. Fitzsimmons, S. D. Bader, J. A. Borchers, G. P. Felcher, J. K. Furdyna, A. Hoffmann, J. B. Kortright, I. K. Schuller, T. C. Schulthess, S. K. Sinha, M. F. Toney, D. Weller, and S. Wolf, J. Magn. Magn. Mat. 271, 103 (2004).

${ }^{26}$ The stray field from the superconducting magnet prevented us from using it with the polarization analyzer. Thus, the polarized beam experiments used a yoked-electromagnet providing a maximum field of $1.2 \mathrm{~T}$.

${ }^{27}$ M. R. Fitzsimmons, S. Park, K. Dumesnil, C. Dufour, R. Pynn, J. A. Borchers, J. J. Rhyne, and P. Mangin, Phys. Rev. B 73, 134413 (2006).

${ }^{28}$ K. Dumesnil, C. Dufour, Ph. Mangin, and A. Rogalev, Phys. Rev. B 65, 094401 (2002).

${ }^{29}$ Since the component of $\vec{Q}$ normal to the sample surface is zero, there is no scattering arising from features as a function of depth into the superlattice.

${ }^{30}$ O. Halpern and M. H. Johnson, Phys. Rev. 55, 898 (1939).

${ }^{31}$ K. L. Krycka, R. A. Booth, C. R. Hogg, Y. Ijiri, J. A. Borchers, W. C. Chen, S. M. Watson, M. Laver, T. R. Gentile, L. R. Dedon, S. Harris, J. J. Rhyne, and S. A. Majetich, Phys. Rev. Lett. 104, 207203 (2010)

${ }^{32}$ W. H. Press et al., Numerical Recipes, The Art of Scientific Computing (Cambridge University Press, Cambridge 1986) p. 470.

${ }^{33}$ M. Sachan et al., Appl. Phys. Lett. 92, 152503 (2008).

${ }^{34}$ G. Grinstein and D. Mukamel, Phys. Rev. B 27, 4503 (1983).

${ }^{35}$ R. L. Leheny, Y. S. Lee, G. Shiane, and R. J. Birgeneau, Eur. Phys. J. B 32, 287 (2003).

${ }^{36}$ F. Hellman, A. L. Shapiro, E. N. Abarra, R. A. Robinson, R. P. Hjelm, P. A. Seeger, J. J. Rhyne, and J. I. Suzuki, Phys. Rev. B 59, 11408 (1999).

${ }^{37}$ T. R. Gentile, G. L. Jones, A. K. Thompson, J. Barker, C. J. Glinka, B. Hammouda, J. W. Lynn, J. Appl. Crystallogr. 33, 771 (2000).

${ }^{38}$ K. L. Krycka, R. Booth, J. A. Borchers, W. C. Chen, C. Conlon, T. R. Gentile, C. Hogg, Y. Ijiri, M. Laver, B. B. Maranville, S. A. Majetich, J. J. Rhyne and S. M. Watson, Physica B (Amsterdam) 404, 2561 (2009). 